\title{
BMJ Open Effects of behavioural parent training for children with attention-deficit/ hyperactivity disorder on parenting behaviour: a protocol for an individual participant data meta-analysis
}

\author{
Laura Steenhuis (D) , ${ }^{1}$ Annabeth P Groenman (D) , ${ }^{1}$ Pieter J Hoekstra, ${ }^{1}$ \\ Rianne Hornstra, ${ }^{1}$ Marjolein Luman, ${ }^{2,3}$ Saskia van der Oord, ${ }^{4}$ \\ Barbara J van den Hoofdakker ${ }^{1}$
}

To cite: Steenhuis $L$, Groenman AP, Hoekstra PJ, et al. Effects of behavioural parent training for children with attention-deficit/ hyperactivity disorder on parenting behaviour: a protocol for an individual participant data meta-analysis. BMJ Open 2020;10:e037749. doi:10.1136/ bmjopen-2020-037749

- Prepublication history and additional material for this paper are available online. To view these files, please visit the journal online (http://dx.doi. org/10.1136/bmjopen-2020037749).

LS and APG contributed equally.

LS and APG are joint first authors.

Received 14 February 2020

Revised 21 August 2020

Accepted 22 September 2020

Check for updates

(C) Author(s) (or their employer(s)) 2020. Re-use permitted under CC BY. Published by BMJ.

For numbered affiliations see end of article.

Correspondence to Dr Annabeth P Groenman; a.groenman@accare.nl

\section{ABSTRACT}

Introduction Behavioural parent training (BPT) is a well-established treatment for children with attentiondeficit/hyperactivity disorder (ADHD). BPT is based on the hypothesis that improvements in parenting are mediators of improvements in children's behaviours. However, metaanalyses show considerate heterogeneity in effects of BPT on child outcomes, and meta-analyses on parenting outcomes are scarce. Also, few studies have investigated parenting factors as mediators of child outcomes. This study aims to examine the effects and moderators of BPT on parenting outcomes and whether improvements in parenting mediate amelioration of behaviour and impairment in children with ADHD.

Methods and analyses We will conduct an individual participant data meta-analysis (IPDMA), making use of individual data of existing trials, and giving the opportunity for highly powered moderator analyses. This IPDMA will be performed by the Psychosocial ADHD INTervention (PAINT) collaboration. We will include randomised controlled trials of BPT, for individuals with ADHD below 18 years old. Systematic searches have been performed to locate relevant papers. Authors are currently contacted to share their data with the PAINT-IPDMA project. We will examine effects of BPT on parenting outcomes (eg, positive and negative parenting, management of affect, perceived parenting competence, parenting stress), moderators of these effects (eg, parental depression, parenting stress, severity of the child's ADHD symptoms) and subsequently perform mediation analyses where parenting outcomes are modelled as mediators of child outcomes (eg, symptoms and severity of ADHD, comorbid psychopathology and impairment).

Ethics and dissemination We will include data from randomised control trials for which ethical approval has been received and consent forms have been signed. Deidentified data will be provided by the original investigators. We aim to disseminate our findings through peer-reviewed scientific journals, presentations at (inter) national scientific meetings, newsletters, the website of our project and the Dutch academic workspace ADHD. PROSPERO registration number CRD42017069877.
Strengths and limitations of this study

- To our knowledge this is the first individual participant data meta-analysis (IPDMA) that examines the effects of behavioural parent training for attentiondeficit/hyperactivity disorder on parenting outcomes.

- By using IPDMA, we are able to conduct highly powered moderation analysis.

- By collecting individual participant data from randomised controlled trials, we have the opportunity to perform uniform analyses across studies.

- Our IPDMA includes only variables that are reported consistently across the majority of studies in the database.

- Representativeness of the database will depend partly on the willingness and ability of investigators to share their data.

\section{INTRODUCTION}

Behavioural parent training (BPT) is a wellestablished intervention for children with attention-deficit/hyperactivity disorder (ADHD) and recommended as first line treatment by many international guidelines. ${ }^{1-3}$ In BPT, parents are trained to apply behavioural techniques meant to increase prosocial and adaptive child behaviours and to reduce disruptive and maladaptive child behaviours. In addition, BPT focusses on nurturing and positive parent-child relationships. ${ }^{4}$ Several meta-analyses on BPT for children with ADHD have shown medium to large effect sizes (ES) on parent-reported reductions of ADHD symptoms, comorbid problems, impairment and parenting behaviours. ${ }^{3}$ 5-7 However, the full range of relevant parenting outcomes of BPT has not been assessed in meta-analyses. ${ }^{7}$ Moreover, while improvements in parenting behaviours are thought 
to mediate improvements in children's behaviours and associated impairments, ${ }^{3}{ }^{9}$ studies investigating these mediation effects are scarce. Another unresolved issue is that individual studies commonly lack the statistical power to adequately assess moderators of improvements of parenting outcomes. Knowing which moderators impact the effectiveness of BPT for child ${ }^{5}$ and parenting outcomes, ${ }^{36}$ in addition to knowing which mediators drive the effects of BPT will give more insight into for whom BPT works best and into the working mechanisms underlying this, therefore contributing to personalised treatment of children with ADHD.

BPT aims to increase the demonstration of positive parenting behaviours and to inhibit negative parenting behaviours, improve the management of affect, reduce parenting stress and enhance parenting self-efficacy, ${ }^{9}$ in order to positively influence children's behaviours and decrease impairment. Parents are trained to modify environmental antecedents (eg, providing structure) and consequences (eg, positive rewards for adaptive behaviour) of behaviour. Positive parenting behaviours consist of providing praise, encouragement, effective communication, demonstrating positive effect and offering physical affirmations, ${ }^{10}{ }^{11}$ whereas negative parenting behaviours are described as providing inconsistent discipline, verbal criticism, corporal punishment, poor supervision and demonstrating negative effect. ${ }^{12-14}$ Essential to positive parenting behaviour is the effective management of parental affect, which is often a specific target of BPT. ${ }^{15}$ Parents are taught to express positive emotions (eg, love, affection and warmth) and to inhibit expressions of negative and unsupportive emotions (eg, anger, frustration and irritability). ${ }^{16}$ Parenting stress (ie, stress arising from the feeling that the demands of parenting outweigh the resources ${ }^{17}$ ) is often reported by parents of children with $\mathrm{ADHD}^{18}$ and is also an important target of BPT. Lastly, parenting self-efficacy is an important target of $\mathrm{BPT}^{19}$; parents should feel more confident and competent in carrying out their parenting tasks.

When assessing parenting outcomes of BPT, it is important to consider a range of outcomes, particularly positive and negative parenting behaviours, management of affect, parenting stress and parenting self-efficacy. However, treatment trials of BPT have not uniformly assessed all parenting outcomes that are explicit targets of BPT, ${ }^{36}$ although BPT outcomes may differ for these different domains. For example, a recent systematic review investigated multiple parenting outcomes (display of parental affect, parenting stress and parenting selfefficacy) of BPT and showed positive results for outcomes most closest to the target of BPT (eg, parenting selfefficacy), but less so for more distal outcomes (eg, parental psychopathology).$^{20}$ Notably, studies in that review were not specifically conducted in ADHD samples, nor were outcomes quantified using a (individual participant data) meta-analytic approach, which will be the analytic approach in the current study. Recently, two meta-analyses examined the effectiveness of behavioural interventions (mostly consisting of BPT) on parenting outcomes in samples of children with ADHD, either assessed by raters unblinded to the treatment condition (eg, parents involved in $\mathrm{BPT}^{9}$ ) or by blinded raters (eg, independent raters coding video-taped interactions between the parent and child $^{36}$ ). The first meta-analysis ${ }^{3}$ considered effects on positive and negative parenting behaviours and parenting self-efficacy, immediately postintervention. Results indicated improvements in positive parenting behaviours (medium ES of 0.68 and 0.63 for unblinded and blinded raters, respectively), reductions in negative parenting behaviours (medium to small ES of 0.57 and 0.43 for unblinded and for blinded raters, respectively) and improvements in parenting self-efficacy (small ES of 0.37 for unblinded raters). The other meta-analysis ${ }^{6}$ investigated the effects of BPT for preschool children with (or at risk for) $\mathrm{ADHD}$, and reported medium to small ES of 0.63 and 0.33 by unblinded and blinded raters, respectively, for reductions in negative parenting behaviours. In addition, long-term effects (up to 12 months) on negative parenting behaviours were reported by unblinded raters with an ES of 0.12 (very small). Overall, both unblinded and blinded parent outcomes show improvements after BPT, although the effects on blinded outcomes generally appeared somewhat smaller than the effects on unblinded outcomes. Improvements on other relevant domains such as parenting stress and display of parental affect have not been addressed in meta-analyses, although individual studies demonstrated that parenting stress can be effectively alleviated with BPT, ${ }^{19}$ and that mothers who participated in BPT have less negative effect and better emotion regulation abilities post-treatment. ${ }^{21}$

Heterogeneity in ES is common in meta-analyses investigating parenting outcomes of BPT for children with ADHD. ${ }^{3}$ More knowledge on factors associated with treatment effects will yield more insight into for whom BPT works best, and may allow clinicians to make better treatment choices tailored to individuals. So far, very few randomised control trials (RCTs) were adequately powered for moderator analyses, and existing moderator analyses were mostly limited to child behavioural outcomes. ${ }^{22-24}$ While traditional methods of addressing heterogeneity in meta-analyses (such as excluding extreme ES, subgroup analyses or meta-regression) may resolve heterogeneity (ie, reduce $\mathrm{I}^{2}$ ), they reveal little about the cause of this heterogeneity. Individual participant data meta-analysis (IPDMA) includes data at an individual level rather than at study level, which enables exploration of moderators, therefore yielding more information about the cause of heterogeneity. Moreover, IPDMA performs a uniform analysis across all studies. IPDMA also has enough power to perform subgroup analyses, which most individual RCTs lack. Due to the collaborative nature of an IPDMA, collaborators can provide input on all phases of the research (including design, analyses, interpretation and manuscript preparation), leading to a high-quality product. $^{25-27}$ So far, no IPDMA has been conducted for parenting outcomes of BPT for children with ADHD. 
In this paper, we present our protocol for an IPDMA on BPT for children with ADHD. The current IPDMA will explore several child-related and parent-related moderators on parenting outcomes of BPT. Given that the examination of moderators on parenting outcomes is scarce, the choice of possible moderators to be investigated in this IPDMA will also be drawn from BPT trials on child outcomes. First, age of the child might be an important moderator, ${ }^{28}$ as parents may have more influence on younger children and younger children may have less engrained symptoms. A meta-analysis indeed demonstrated that BPT had more effect on positive parenting behaviours for younger compared with older children with ADHD. ${ }^{3}$ Second, medication use of the child may moderate BPT outcomes, as parents of children on medication might find it easier to adapt their parenting styles and experience less resistance when doing so, although there could also be a floor effect as that children with medication might already function better. Previous studies suggested that medication use may positively contribute to BPT outcomes on child's ADHD symptoms, ${ }^{29}$ although results have been mixed. ${ }^{22}$ Third, it seems plausible that intelligence of the child is positively associated with a treatment that involves learning associations between behaviours and consequences and new skills. ${ }^{30}$ There is some evidence that higher children's Intelligence Quotient (IQ) is associated with more improvement in ADHD symptoms following behavioural treatment, but only for specific subgroups of children, such as girls with more antisocial symptoms. ${ }^{24} 3132$ Fourth, pretreatment ADHD severity and presence of comorbidities could moderate BPT, as parents might find it easier to change their parenting behaviour when the child has less complex symptomatology. Previous individual studies have indeed confirmed that comorbidities at baseline negatively impacted the outcome of BPT treatment with regard to child symptoms. ${ }^{22} 2333$

There are several parent-related moderators that may have an effect on parenting outcomes of BPT. First, parental mental health problems (depression, ADHD, parental stress) are likely to affect how well parents are able to grasp new information and impact their ability to learn new methods of parenting behaviours and skills. 2223 34-36 Second, low socioeconomic status (SES) may moderate outcomes of BPT, as this may increase family strain and impact the availability of family resources. There is mixed evidence of the moderating effect of low SES on the effects of BPT on ADHD symptoms and related problems, showing either no effect, ${ }^{24}$ or better outcomes for parents with lower SES. ${ }^{37}$ So far, effects of low SES in relation to parenting outcomes of BPT for children with ADHD have not been investigated. For the present IPDMA we will rely on an imperfect measure of SES, that is, parental education, as we are reliant on the measures that are consistently used across studies Third, single parenthood may also moderate outcomes of BPT, as individual studies have demonstrated that single mothers are less likely to respond to $\mathrm{BPT}^{38}{ }^{39}$ and maintain treatment gains over time $^{40}$ in terms of parenting behaviour. Indeed, research suggests that single parents benefit more from enhanced BPT programmes, suited to their specific needs, ${ }^{41}{ }^{42}$ compared with standard BPT programmes. Fourth, there is evidence that lower parenting self-efficacy at baseline has a negative impact on improvements in behavioural problems in children with ADHD following BPT $^{43}$ and one could expect similar results for parenting outcomes. On the other hand, it is also possible that lower parenting abilities at baseline yield more room for improvement in BPT, and thus both directions of the effect could be expected.

Following the investigation of parenting outcomes of BPT as part of our IPDMA, the next step will be to examine mediators of improvements in child's behaviour following BPT. Currently, there are no meta-analyses analysing whether improvements in parenting behaviour mediate improvements of BPT on symptoms of ADHD of the child. Some evidence of individual studies shows that reductions in parenting behaviours or parenting attributions mediate improvements on child outcomes in behavioural interventions. ${ }^{12} 3544$ This IPDMA aims to synthesise the available data regarding the association between improvements in parent outcomes and improvements in child's outcomes following BPT.

The specific aims of our IPDMA include:

1. To investigate effects of BPT on parenting outcomes (positive and negative parenting behaviours, display of parental affect, parenting stress and parenting selfefficacy). Given that BPT for ADHD has shown different effects when assessed by unblinded raters and by more blinded raters, ${ }^{367}$ we aim to distinguish (if possible) between unblinded and blinded assessments of parenting behaviour and display of parental affect.

2. To investigate possible child (including age, medication use, IQ, ADHD severity, presence and severity of comorbidities) and parent (including depression, ADHD, SES, single parenthood and parenting measures) moderators of parenting outcomes.

3. To investigate whether improvements in parenting behaviours mediate the effect of BPT on behavioural child outcomes and impairment following treatment.

For aims 1-3, outcomes of BPT will be examined immediately post-treatment and at long-term where possible.

\section{METHODS AND ANALYSIS}

For this IPDMA we will build on the Psychosocial ADHD INTervention IPD (PAINT-IPD) database which is registered in PROSPERO (https://www.crd.york.ac.uk/PROSPERO/display_record.php? ID=CRD42017069877\&ID= CRD42017069877). In this project, we collect data on psychosocial treatments for children with ADHD: the search is regularly updated and the database continues to expand. For the purpose of the current IPDMA on parenting outcomes, we will use the same methods and search strategy. This protocol is written in line with the Preferred Reporting Items for Systematic Reviews and 
Meta-Analysis-Protocols (PRISMA-P) 2015 checklist ${ }^{45}$ (see online supplemental checklist). The current study is planned to commence in September 2020.

\section{Inclusion criteria}

We will include RCTs of behavioural treatments of individuals aged below 18 with ADHD, corroborated by clinical cutoffs on questionnaires or (semi)-structured interviews. We will include studies that compared BPT with a control condition (ie, all conditions that are labelled control, including active treatment), and studies that compared BPT to another behavioural intervention (head to head comparisons). We excluded studies or intervention arms that used optimised medication treatment next to BPT as part of their study design or as a control condition. We define BPT as interventions directed at changing children's behaviours (ie, increasing desirable behaviours and decreasing undesirable behaviours), using (cognitive) behavioural therapeutic techniques which parents are trained in. ${ }^{46}$ Multimodal interventions (consisting of both parent and/or teacher and/or child training), will be included if the time spent on parent training within the intervention was at least equal to other types of training.

\section{Selection and screening of studies}

The last systematic search was performed on 13 May 2020. Currently, we are contacting the authors of the newly identified studies. Two authors (APG and RH) performed the selection and screening of studies, disagreement was resolved by consensus. A two-step approach to identifying relevant articles was used. First, Medline, CINAHL, PsycINFO, EMBASE+EMBASE CLASSIC, ERIC, Web of Science (Science Citation Index Expanded) was searched for relevant papers using a combination of the following search terms and their synonyms, as well as hierarchical family form (eg, MeSH terms): treatment-specific terms (eg, behavioural treatment, psychosocial treatment and parent training), ADHD, child and RCT. No date restrictions were applied. English, German and Dutch language publications published in peer-reviewed journals were included. Second, literature lists of all selected studies and relevant systematic reviews and meta-analyses were handsearched to identify possible missing articles (complete search criteria for each database are available in online supplemental 1.

\section{Data collection and management \\ Author contact}

We will contact the corresponding authors of all eligible trials to ask for their participation in the current IPD metaanalyses. If after several weeks we have had no response, we will send a reminder to the corresponding author. If we have failed to establish contact with the corresponding author, we will email the other authors of the study. Furthermore, we will contact researchers during conferences, and through our personal network to retrieve all eligible databases.

\section{Data format and management}

Part of the data has already been received in light of our previous IPDMA. ${ }^{5}$ For newly identified data that we have not yet received, we will use the best, safest way to transfer the data. The most convenient way for most authors will be to transfer the encrypted data per email to the project management, but we will be open to other options (eg, face to face transfer). A copy of our data collection manual for the PAINT-IPD database can be found in online supplemental 2). We will allow authors to send the data in all possible formats, although the most preferable format would be one in which each subject represents a row and each variable a column.

If authors are not included in the PAINT collaborators group yet, ${ }^{5}$ we will offer one or two authors of each included study a place in our PAINT collaborators group. We plan yearly telephone calls and/or meetings at large international conferences to keep the working group up to date and discuss design and methodological issues. Members of the working group who have provided data on BPT trials with parenting outcomes will have the opportunity to provide feedback on the first draft of the manuscript and will be sent a copy of the final manuscript before submission. Only authors in the IPD steering committee group (APG, BJvdH, SvdO, ML and PJH) will have access to the data.

\section{Ethics and dissemination}

The original investigators will be asked for de-identified data, so that only the original investigator knows the link between data and participant. We will only include RCTs where ethical approval has been given and participants signed consent forms. Since the current IPDMA is an extension of the original purpose of the eligible studies, we do not expect any ethical issues with the current IPDMA. Results of our study will be disseminated through peer-reviewed scientific journals, and presentations on (inter)national scientific and/or clinical expert meetings.

\section{Patient and public involvement}

An expert panel consisting of parents of children with ADHD was organised at the conception of the idea in which feedback was given to the plans and changes were made accordingly. Our results will be communicated to clinicians, clients and their parents through newsletters and through the academic workplace 'ADHD en druk gedrag' (also see https://adhdendrukgedrag.nl/) in which many parents, client organisations and mental healthcare professionals are represented.

\section{Variables}

For the PAINT-IPD database, a data request form will be sent to all authors of the original studies, containing a list of variables that will be requested (see online supplemental 2a). This list was determined by reviewing the literature and the IPDMA steering committee (authors APG, SvdO, ML, PJH and BJvdH) assessed these outcomes 
domains for suitability and interest. The final list of variables will depend on the available data of all studies.

For the current protocol, the following parenting variables will be selected:

\section{Parenting variables}

Positive and negative parenting behaviours will be assessed using unblinded and blinded measures. For unblinded parenting measures, we will select questionnaires such as the Alabama Parenting Questionnaire. ${ }^{10}$ For blinded measures, we will select assessments used to observe and code parent behaviour, such as the Dyadic Parent-Child Interaction Coding System. ${ }^{47}$

Display of positive and negative parental affect will be assessed with measures specifically designed to capture parental affect (eg, 5-minute speech sample of expressed emotion ${ }^{15}$;) or a subscale of an existing parenting scale (eg, attachment domain of the Parenting-Relationship Questionnaire ${ }^{48}$ ).

Parental stress will be assessed using measures specifically designed to capture stress from parenting, such as the Parenting Stress Index ${ }^{49}$, but also measures assessing stress in the caregiver more generally (Depression and Anxiety Stress Scales (DASS ${ }^{50}$ ).

Parenting self-efficacy will be assessed using measures specifically designed to capture parenting self-efficacy, such as the Parental Sense of Competence Scale, ${ }^{51}$ a subscale of an existing parenting scale (eg, the ParentingRelationship Questionnaire ${ }^{48}$ ) or a scale designed to assess parenting competence in specific contexts, such as education (eg, Parent as Educator Scale ${ }^{52}$ ).

Parental depression will be assessed using measures to capture depressive symptoms, such as the Beck Depression Inventory ${ }^{53}$, a subscale of an existing mental health questionnaire (eg, depression subscale of the DASS ${ }^{54}$ ) or a mental health questionnaire for which the overall score can be used as a proxy for depression (eg, the General Health Questionnaire ${ }^{55}$ ).

Parental ADHD will be assessed using an adult measure of ADHD, such as the Adult Self-Report Scale Screener. ${ }^{56}$

The remaining parent variables, including single parenthood and SES, are commonly assessed with demographic questionnaires or items. SES can be assessed in different ways (eg, based on income, occupation, neighbourhood or education) and due to our IPDMA methodology, we are reliant on choosing the assessment measure that is most consistently used across studies. Given that data on parental education is often available, and is deemed acceptable as a proxy for SES, ${ }^{5}$ parental education level will serve as a proxy measure for SES in the current study. SES will be conceptualised as low (<high school), medium (high school graduate or college education) or high (>college graduate).

Note that parenting behaviours, parental affect, parental stress and parenting self-efficacy will serve as moderators (to predict parenting outcomes), mediators (in the relationship between BPT and child outcomes) and outcome variables (in the moderator analysis). Parental depression, parental ADHD, single parenthood and SES will serve as baseline moderators to predict parenting outcomes.

\section{Child variables}

Additionally, the following child variables will be selected:

Child ADHD severity will be assessed using a parent-rated measure of childhood ADHD, such as the ADHD subscale of the Connors Parent Rating Scale. ${ }^{57}$

Comorbidity will be assessed using symptoms of oppositional defiant disorder (ODD) and/or conduct disorder (CD), by for example the ODD subscale of the Connors Parent Rating Scale, ${ }^{57}$ or the CD subscale of the Disruptive Behaviour Disorder Rating Scale ${ }^{58}$ In addition, internalising symptoms will be assessed, using for example the internalising subscale of the Child Behaviour Checklist. ${ }^{59}$

Global impairment will be assessed using a parent-rated or clinician-rated measure of global impairment, such as the Impairment Rating Scale. ${ }^{60}$

The remaining child variables, including child age, medication use and $I Q$ are commonly assessed with demographic questionnaires or items.

Child ADHD severity, comorbidity and global impairment will serve as moderators (to predict parenting outcomes) and outcome variables (in the mediation analysis). Child age, medication use and $I Q$ will solely serve as moderators to predict parenting outcomes.

For all variables (moderators and outcomes), when a study reports multiple measures to capture the same concept, the measure which is most often used by other studies will be included. If multiple raters (mothers and fathers) are included to assess the same concept, the assessment by the mother will be preferred as they are more often the primary caregiver and more often take part in BPT. Regarding harmonisation, for each dataset continuous measures will be converted into z-scores, using pre-intervention-score SD within studies.

\section{Quality assessment}

Quality assessment will be done independently by three authors (a combination of APG, RH and LS) using Cochrane risk of bias. Any disagreement will be resolved by consensus. Once the data has been received, all raw data sets will be checked for impossible, missing or extreme values. We will collect data on all randomised participants. As this will possibly reintroduce participants who were previously excluded, we will also check randomisation parameters (eg, age, sex and ADHD severity of the participants). If any unexpected deviations are found between our results and the published results, the original researcher will be contacted to locate the origin of this deviation.

\section{Analysis}

Effects of BPT will be calculated using a one-stage IPDMA, in which data from participants across studies will be analysed in one stage, clustered by study. A linear multilevel analysis will be used to examine the effects of BPT on parenting outcome measures. A random intercept 
for study will be added to each model. Post intervention outcome measures will be used as dependent variables in these models, and preintervention outcome measures and intervention group will be added. The interaction between intervention group and moderators of interest will be added to the model to assess their moderating effect on treatment outcomes. To analyse changes in parenting behaviours as a potential mediator in the relationship between BPT and child outcomes, the change in parenting behaviours (post-treatment score - baseline score) will be used. An interaction between intervention group and change in parenting behaviours will be added to the model to determine whether change in parenting behaviours had a main effect on child outcomes and/ or an interactive effective with treatment. ${ }^{61}{ }^{62}$ We will conduct sensitivity analyses between studies that provided data and those that did not, for demographic characteristics (eg, age) and for inclusion criteria (cut-off on measures vs meeting diagnostic criteria for ADHD) and reported ES. If sufficient data is available, a subgroup analysis for child age will be conducted, where all analyses will be repeated separately for pre-school children $(<6$ years of age), school-aged children (6-12 years of age) and adolescents ( $>12$ years of age).

\section{DISCUSSION}

The current protocol presents the first IPDMA to synthesise research findings on treatment effects of BPT for children with ADHD on parenting outcomes. Both child and parent moderators of parenting outcomes will be explored and a mediation analysis will be conducted to examine whether changes in parenting behaviours mediate the effect of BPT on child behaviours and impairment. Previous efforts to identify moderators of parenting outcomes are scarce, as there are few well-powered RCTs and only two meta-analyses on this topic. ${ }^{36}$ The heterogeneity found in parenting outcomes in previous metaanalyses suggests that there is not a 'one size fits all' solution of BPT for children with ADHD. Parents and children who differ with regard to their personal, clinical and demographic characteristics, are also likely to differ in their response to BPT. Clearly, an IPDMA approach is needed to further examine the effects of BPT on parenting outcomes, to elucidate potential sources of heterogeneity among children and parents, and to investigate potential mechanisms of change in BPT for children with ADHD. Ultimately, both research and clinical practice may be informed by the knowledge of which child and/or parent responds best to a certain treatment, thereby contributing to the overall goal of providing the best care to children with ADHD and their parents.

An additional important aim of the current IPDMA will be to examine a crucial assumption about the working mechanism of BPT, namely whether improvements in parenting behaviours mediate improvements in child behaviour and impairment. So far, single studies have examined this assumption, finding some supportive evidence, ${ }^{12} 19$ but this hypothesis has never been addressed in a meta-analysis, let alone by using an IPDMA approach. By synthesising all available (raw) data from RCTs, we will conduct highly sophisticated and powered statistical analyses, to provide insight into the working mechanisms of BPT. This knowledge will allow us to further improve and refine BPT programmes for children with ADHD.

Despite clear strengths of the IPDMA approach, such as high-powered moderator, mediation and subgroup analyses, there are some limitations as well. ${ }^{25} 2763$ First, IPDMA cannot change anything about the way the study was originally conducted. Second, it is unclear whether enough studies measured the variables of interest, and thus whether all intended outcomes can be examined. Third, if investigators of trials are unable to share their data (or may not be willing to share their data), not all relevant data can be included in the synthesis. Fourth, not all factors which may affect study outcomes can be conceptualised as a moderator, such as efficacy versus effectiveness trials (ie, studies often do not fit exactly into one of both categories). Fifth, since parental education levels are easy to collect, they are omnipresent in the data. Ideally, several aspects of SES are taken into account, such as income, occupation, and/or neighbourhood, ${ }^{6465}$ but this data is available in only a few datasets.

Notwithstanding these limitations, this study has the potential to elucidate clinically relevant questions concerning the efficacy of BPT for children with ADHD and their parents and to provide insight in moderators and mediators of treatment effects. This knowledge may improve and optimise current treatment programmes and could eventually lead to advances in personalised treatment for children with ADHD.

\section{Author affiliations}

${ }^{1}$ Department of Child and Adolescent Psychiatry, University of Groningen, University Medical Center Groningen, Groningen, the Netherlands

${ }^{2}$ Dept. Clinical Neuropsychology, Vrije Universiteit Amsterdam, Amsterdam, The Netherlands

${ }^{3}$ Bascule, academic centre for child and adolescent psychiatry, Amsterdam, the Netherlands

${ }^{4}$ Clinical Psychology, KU Leuven, Leuven, Flanders, Belgium

Acknowledgements The authors would like to thank all collaborators of the PAINT-IPDMA project for providing input to this protocol and for providing data for this project. The authors thank our parents of clients' expert panel for their valuable input during the conception of the idea for the PAINT-IPDMA.

Contributors BJvdH is the guarantor. APG wrote the first draft of the manuscript, and $L S$ wrote a revised and final version. APG, RH and LS are performing the systematic search and data extraction. PJH, ML, Svd0 and BJvdH acquired funding for the PAINT-IPDMA project; an international database including individual participant data from RCTs examining psychosocial interventions for the treatment of ADHD in children. The current study is a part of the PAINT-IPDMA project. PJH, ML, Svd0, BJvdH and APG setup the initial design for the IPDMA. All authors worked together to design this IPDMA, contributed to the intellectual content of the current manuscript and approved the final version.

Funding This research was funded by The Dutch Organization for Health Research and Development (ZonMw) under grant number 729300013.

Disclaimer The funder had no role in the design of this protocol. The funder will have no input on the collection of data, the data analysis or the interpretation or publication of the study results. 
Competing interests None declared.

Patient consent for publication Not required.

Provenance and peer review Not commissioned; externally peer reviewed.

Supplemental material This content has been supplied by the author(s). It has not been vetted by BMJ Publishing Group Limited (BMJ) and may not have been peer-reviewed. Any opinions or recommendations discussed are solely those of the author(s) and are not endorsed by BMJ. BMJ disclaims all liability and responsibility arising from any reliance placed on the content. Where the content includes any translated material, BMJ does not warrant the accuracy and reliability of the translations (including but not limited to local regulations, clinical guidelines, terminology, drug names and drug dosages), and is not responsible for any error and/or omissions arising from translation and adaptation or otherwise.

Open access This is an open access article distributed in accordance with the Creative Commons Attribution 4.0 Unported (CC BY 4.0) license, which permits others to copy, redistribute, remix, transform and build upon this work for any purpose, provided the original work is properly cited, a link to the licence is given, and indication of whether changes were made. See: https://creativecommons.org/ licenses/by/4.0/.

\section{ORCID iDs}

Laura Steenhuis http://orcid.org/0000-0002-1256-3754

Annabeth P Groenman http://orcid.org/0000-0002-8394-6605

\section{REFERENCES}

1 Wolraich M, et al, Subcommittee on Attention-Deficit/Hyperactivity Disorder, Steering Committee on Quality Improvement and Management. ADHD: clinical practice guideline for the diagnosis, evaluation, and treatment of attention-deficit/hyperactivity disorder in children and adolescents. Pediatrics 2011;128:1007-22.

$2 \mathrm{NICE} \mathrm{NI} \mathrm{for} \mathrm{H}$ and $\mathrm{CE}$. Attention deficit hyperactivity disorder: diagnosis and management (NICE guideline 87). Natl Inst Heal Care Excell 2018.

3 Daley D, van der Oord S, Ferrin M, et al. Behavioral interventions in attention-deficit/hyperactivity disorder: a meta-analysis of randomized controlled trials across multiple outcome domains. J Am Acad Child Adolesc Psychiatry 2014;53:835-47.

4 Kazdin AE. Parent management training: evidence, outcomes, and issues. J Am Acad Child Adolesc Psychiatry 1997:36:1349-56.

5 Groenman AP, Hornstra R, Hoekstra PJ, et al. Behavioral treatments for children and adolescents with attention-deficit/hyperactivity disorder: an individual participant data meta-analysis. Submitted.

6 Rimestad ML, Lambek R, Zacher Christiansen H, et al. Short- and long-term effects of parent training for preschool children with or at risk of ADHD: a systematic review and meta-analysis. J Atten Disord 2019;23:423-34.

7 Sonuga-Barke EJS, Brandeis D, Cortese S, et al. Nonpharmacological interventions for ADHD: systematic review and meta-analyses of randomized controlled trials of dietary and psychological treatments. Am J Psychiatry 2013;170:275-89.

8 Mulqueen JM, Bartley CA, Bloch MH. Meta-Analysis: parental interventions for preschool ADHD. J Atten Disord 2015;19:118-24.

9 Daley D, Van Der Oord S, Ferrin M, et al. Practitioner review: current best practice in the use of parent training and other behavioural interventions in the treatment of children and adolescents with attention deficit hyperactivity disorder. J Child Psychol Psychiatry 2018;59:932-947:932-47.

10 Frick P. The Alabama parenting questionnaire. Unpubl Rat scale, Univ Alabama 1991.

11 Morris AS, Silk JS, Steinberg L, et al. Temperamental vulnerability and negative parenting as interacting predictors of child adjustment. $J$ Marriage Fam 2002.

12 Hinshaw SP, Owens EB, Wells KC, et al. Family processes and treatment outcome in the MTA: negative/ineffective parenting practices in relation to multimodal treatment. $J$ Abnorm Child Psychol 2000;28:555-68.

13 Sanders MR. Triple P-Positive parenting program as a public health approach to strengthening parenting. J Fam Psychol 2008;22:506-17.

14 Wells KC, Epstein JN, Hinshaw SP, et al. Parenting and family stress treatment outcomes in attention deficit hyperactivity disorder. $J$ Abnorm Child Psychol 2000;6:543-53 https://link.springer.com/ content/pdf/10.1023\%2FA\%3A1005131131159.pdf

15 Daley D, Sonuga-Barke EJS, Thompson M. Assessing expressed emotion in mothers of preschool AD/HD children: psychometric properties of a modified speech sample. Br J Clin Psychol 2003;42:53-67.

16 Herbert SD, Harvey EA, Roberts JL, et al. A randomized controlled trial of a parent training and emotion socialization program for families of hyperactive preschool-aged children. Behav Ther 2013;44:302-16.

17 Deater-Deckard K, Smith J, Ivy L, et al. Differential perceptions of and feelings about sibling children: implications for research on parenting stress. Infant Child Dev 2005

18 Johnson JH, Reader SK. Assessing stress in families of children with ADHD: preliminary development of the disruptive behavior stress inventory (DBSI). J Clin Psychol Med Settings 2002.

19 Heath CL, Curtis DF, Fan W, et al. The association between parenting stress, parenting self-efficacy, and the clinical significance of child ADHD symptom change following behavior therapy. Child Psychiatry Hum Dev 2015;46:118-29.

20 Colalillo S, Johnston C. Parenting cognition and affective outcomes following parent management training: a systematic review. Clin Child Fam Psychol Rev 2016;19:216-35.

21 Gershy N, Meehan KB, Omer H, et al. Randomized clinical trial of mindfulness skills augmentation in parent training. Child Youth Care Forum 2017;46:783-803.

22 van den Hoofdakker BJ, Nauta MH, van der Veen-Mulders L, et al. Behavioral parent training as an adjunct to routine care in children with attention-deficit/hyperactivity disorder: moderators of treatment response. J Pediatr Psychol 2010;35:317-26.

23 Veenman B, Luman M, Oosterlaan J. Moderators influencing the effectiveness of a behavioral teacher program. Front Psychol 2018;9:298

24 Owens EB, Hinshaw SP, Arnold LE, et al. Which treatment for whom for ADHD? Moderators of treatment response in the MTA. J Consult Clin Psychol 2003

25 Riley RD, Lambert PC, Abo-Zaid G. Meta-Analysis of individual participant data: rationale, conduct, and reporting. $B M J$ 2010;340:c221.

26 Higgins JPT, Jackson D, Barrett JK, et al. Consistency and inconsistency in network meta-analysis: concepts and models for multi-arm studies. Res Synth Methods 2012;3:98-110.

27 Stewart LA, Tierney JF. To IPD or not to IPD? advantages and disadvantages of systematic reviews using individual patient data. Eval. Heal. Prof 2002.

28 Owens EB, Hinshaw SP, McBurnett K, et al. Predictors of response to behavioral treatments among children with ADHD-Inattentive type. $J$ Clin Child Adolesc Psychol 2018;47:S219-32.

29 Jensen A, Richters S, al VV. Moderators and mediators of treatment response for children with attention-deficit/hyperactivity disorder: the multimodal treatment study of children with attention-deficit/ hyperactivity disorder. Arch Gen Psychiatry 1999;56:1088-96.

30 Hallahan DP. Cognitive-Behavioral therapy for impulsive children. Child Youth Serv Rev 1986.

31 Kazdin AE, Crowley MJ. Moderators of treatment outcome in cognitively based treatment of antisocial children. Cognit Ther Res 1997;21:185-207.

32 Van der Oord S, Prins PJM, Oosterlaan J, et al. Efficacy of methylphenidate, psychosocial treatments and their combination in school-aged children with ADHD: a meta-analysis. Clin Psychol Rev 2008;28:783-800.

33 Lee SS, Falk AE, Aguirre VP. Association of comorbid anxiety with social functioning in school-age children with and without attentiondeficit/hyperactivity disorder (ADHD). Psychiatry Res 2012.

34 Langberg JM, Evans SW, Schultz BK, et al. Trajectories and predictors of response to the challenging horizons program for adolescents with ADHD. Behav Ther 2016;47:339-54.

35 Chronis-Tuscano A, O'Brien KA, Johnston C, et al. The relation between maternal ADHD symptoms \& improvement in child behavio following brief behavioral parent training is mediated by change in negative parenting. J Abnorm Child Psychol 2011;39:1047-57.

36 Sonuga-Barke EJS, Daley D, Thompson M. Does maternal ADHD reduce the effectiveness of parent training for preschool children's ADHD? J Am Acad Child Adolesc Psychiatry 2002;41:696-702.

37 Farmer CA, Brown NV, Gadow KD, et al. Comorbid symptomatology moderates response to risperidone, stimulant, and parent training in children with severe aggression, disruptive behavior disorder, and attention-deficit/hyperactivity disorder. J Child Adolesc Psychopharmacol 2015;25:213-24.

38 Dumas JE, Wahler RG. Predictors of treatment outcome in parent training: mother insularity and socioeconomic disadvantage. Behav Assess 1983.

39 Webster-Stratton C, Hammond M. Predictors of treatment outcome in parent training for families with conduct problem children. Behav Ther 1990. 
40 Webster-Stratton C. Predictors of treatment outcome in parent training for conduct disordered children. Behav Ther 1985.

41 Chacko A, Wymbs BT, Wymbs FA, et al. Enhancing traditional behavioral parent training for single mothers of children with ADHD. $J$ Clin Child Adolesc Psychol 2009;38:206-18.

42 Chacko A, Uderman JZ, Zwilling A. Lessons learned in enhancing behavioral parent training for high-risk families of youth with ADHD. ADHD Rep 2013;21:11:6-11.

43 van den Hoofdakker BJ, Hoekstra PJ, van der Veen-Mulders L, et al. Paternal influences on treatment outcome of behavioral parent training in children with attention-deficit/hyperactivity disorder. Eur Child Adolesc Psychiatry 2014;23:1071-9.

44 Booster GD, Mautone JA, Nissley-Tsiopinis J, et al. Reductions in negative parenting practices mediate the effect of a family-school intervention for children with attention deficit hyperactivity disorder School Psych Rev 2016.

45 Shamseer L, Moher D, Clarke M, et al. Preferred reporting items for systematic review and meta-analysis protocols (PRISMA-P) 2015: elaboration and explanation. BMJ 2015;349:97647.

46 Evans SW, Owens JS, Bunford N. Evidence-Based psychosocial treatments for children and adolescents with attention-deficit/ hyperactivity disorder. J Clin Child Adolesc Psychol 2014.

47 Eyberg SM, Nelson MMD, Duke M, et al. Manual for the Dyadic parent-child interaction coding system. 3rd ed. System, 2004.

48 Rubinic D, Schwickrath H, Test Review: Kamphaus RW, et al. Parenting relationship questionnaire. Minneapolis, Mn: NCS Pearson. J Psychoeduc Assess 2006;2010.

49 Abidin RR. Parenting stress and the utilization of pediatric services. Children's Health Care 1982;11:70-3.

50 Clara IP, Cox BJ, Enns MW. Confirmatory factor analysis of the Depression-Anxiety-Stress scales in depressed and anxious patients. $J$ Psychopathol Behav Assess 2001;23:61-7.

51 Ohan JL, Leung DW, Johnston C. The parenting sense of competence scale: evidence of a stable factor structure and validity. Can J Behav Sci 2000.

52 Mautone JA, Marcelle E, Tresco KE, et al. Assessing the quality of parent-teacher relationships for students with ADHD. Psychol Sch 2015;52:196-207.
53 Beck AT, Steer RA, Carbin MG. Psychometric properties of the Beck depression inventory: twenty-five years of evaluation. Clin Psychol Rev 1988

54 Henry JD, Crawford JR. The short-form version of the depression anxiety stress scales (DASS-21): construct validity and normative data in a large non-clinical sample. Br J Clin Psychol 2005;44:227-39.

55 Goldberg DP, Hillier VF. A scaled version of the general health questionnaire. Psychol Med1979.

56 Kessler RC, Adler L, Ames M, et al. The world Health organization adult ADHD self-report scale (ASRS): a short screening scale for use in the general population. Psychol Med 2005

57 Kao GS, Thomas HM, Test Review: C. Keith Conners Conners 3rd edition Toronto, Ontario, Canada: Multi-Health systems, 2008. J Psychoeduc Assess 2010

58 WE PJ, Gnagy EM, Greenslade KE, et al. Teacher ratings of DSM-III-R symptoms for the disruptive behavior disorders. [erratum appears in J Am Acad Child Adolesc Psychiatry 1992 Nov;31(6):1177]. J Am Acad Child Adolesc Psychiatry1992.

59 Achenbach TM. Manual for the Youth Self Report and 1991 Profile, 1991.

60 Fabiano GA, Pelham WE, Waschbusch DA, et al. A practical measure of impairment: psychometric properties of the impairment rating scale in samples of children with attention deficit hyperactivity disorder and two school-based samples. $J$ Clin Child Adolesc Psychol 2006;35:369-85.

61 Kraemer HC, Wilson GT, Fairburn CG, et al. Mediators and moderators of treatment effects in randomized clinical trials. Arch Gen Psychiatry 2002;59:877-83.

62 Haasova M, Warren FC, Ussher M, et al. The acute effects of physical activity on cigarette cravings: exploration of potential moderators, mediators and physical activity attributes using individual participant data (IPD) meta-analyses. Psychopharmacology 2014;231:1267-75.

63 Higgins JP, Whitehead A, Turner RM, et al. Meta-Analysis of continuous outcome data from individual patients. Stat Med 2001;20:2219-41.

64 Shavers VL. Measurement of socioeconomic status in health disparities research. J Natl Med Assoc 2007;99:1013-23.

65 Braveman PA, Cubbin C, Egerter S, et al. Socioeconomic status in health research: one size does not fit all. J Am Med Assoc 2005. 